\title{
Avaliação Hemodinâmica e Metabólica da Infusão Contínua de Dexmedetomidina e de Remifentanil em Colecistectomia Videolaparoscópica. Estudo Comparativo *
}

\author{
Hemodynamic and Metabolic Evaluation of Dexmedetomidine and \\ Remifentanil Continuous Infusion in Videolaparoscopic \\ Cholecystectomy. Comparative Study
}

Thatiany Pereira Chaves ${ }^{1}$; Josenília Maria Alves Gomes, TSA ${ }^{1}$; Francisco Elano Carvalho Pereira ${ }^{1}$; Sara Lúcia Cavalcante, TSA ${ }^{2}$; IIse M. Tigre de Arruda Leitão ${ }^{3}$; Hipólito Sousa Monte ${ }^{4}$; Rodrigo Dornfeld Escalante ${ }^{5}$

\begin{abstract}
RESUMO
Chaves TP, Gomes JMA, Pereira FEC, Cavalcante SL, Leitão IMTA, Monte HS, Escalante RD - Avaliação Hemodinâmica e Metabólica da Infusão Contínua de Dexmedetomidina e de Remifentanil em Colecistectomia Videolaparoscópica. Estudo Comparativo
\end{abstract}

JUSTIFICATIVA E OBJETIVOS: A dexmedetomidina tem sido utilizada para sedação e como coadjuvante em anestesia geral. O objetivo deste estudo foi avaliar a resposta cardiovascular e simpático-adrenal à intubação traqueal e à insuflação do pneumoperitônio, comparando-a ao remifentanil durante anestesia com sevoflurano para colecistectomia videolaparoscópica.

MÉTODO: Foram incluídos no estudo 42 pacientes, estado físico ASA I ou II, com idades entre 25 e 55 anos, distribuídos aleatoriamente em dois grupos: GI e Gll. A indução da anestesia foi realizada com infusão contínua de $1 \mu \mathrm{g} . \mathrm{kg}^{-1}$ de dexmedetomidina (GI) ou remifentanil (GII), durante 10 minutos, seguido de propofol e cisatracúrio. A manutenção da anestesia foi realizada com a infusão contínua de 0,7 $\mu \mathrm{g} \cdot \mathrm{kg}^{-1} \cdot \mathrm{h}^{-1}$ de dexmedetomidina ou $0,5 \mu \mathrm{g} \cdot \mathrm{kg}^{-1} \cdot \mathrm{h}^{-1}$ de remifentanil e concentrações variadas de sevoflurano. Foram anotadas a PAS, PAD e FC nos momentos: $M_{1}$ - antes do início da infusão inicial da droga; $M_{2}$ - após término da infusão inicial da droga; $M_{3}$ - após a intubação orotraqueal; $M_{4}$ - antes do início do pneumoperitônio; $M_{5}$ - após o pneumoperitônio; $M_{6}$ cinco minutos após desinsuflado o pneumoperitônio, $M_{7}$ - após extubação traqueal. Em $M_{4}, M_{5}$ e $M_{6}$ foram dosadas adrenalina e noradrenalina. A concentração expirada (CE) do sevoflurano, a relação CE/CAM, consumo de sevoflurano foram registrados em $M_{4}, M_{5}$ e $M_{6}$.

RESULTADOS: Variações na PAS e PAD foram maiores no grupo da dexmedetomidina em $M_{4}$ a $M_{5}$. A FC e os níveis de adrenalina e noradrenalina não apresentaram diferença entre os grupos. A CE do sevoflurano foi maior em $M_{4}$ e $M_{6}$ no $\mathrm{Gl}$, assim como a CE/CAM. No GI, o consumo de sevoflurano foi

\footnotetext{
* Recebido do (Received from) Serviço de Anestesiologia do Hospital Monte Klinikum, Fortaleza, CE

1. Anestesiologista do Hospital Monte Klinikum

2. Professora da UFC

3. Enfermeira do Hospital Monte Klinikum

4. Diretor do Hospital Monte Klinikum

5. Cirurgião do Hospital Monte Klinikum

Endereço para correspondência (Correspondence to)

Dra. Thatiany Pereira Chaves

Rua Pereira de Miranda, 955 / 601 Papicu

60175-045 Fortaleza, CE
}

Apresentado (Submitted) em 22 de outubro de 2002

Aceito (Accepted) para publicação em 09 de dezembro de 2002

(C) Sociedade Brasileira de Anestesiologia, 2003 maior e observou-se uma tendência para menor consumo de analgésicos e antieméticos.

CONCLUSÕES: Nas condições deste estudo, a dexmedetomidina inibiu a liberação de catecolaminas durante a intubação orotraqueal e o pneumoperitônio, porém, não impediu o aumento da pressão arterial em resposta à insuflação peritoneal.

Unitermos: ANALGÉSICOS, Opióides: remifentanil; CIRURGIA, Videolaparoscópica: colecistectomia; HIPNÓTICOS: dexmedetomidina

\section{SUMMARY}

Chaves TP, Gomes JMA, Pereira FEC, Cavalcante SL, Leitão IMTA, Monte HS, Escalante RD - Hemodynamic and Metabolic Evaluation of Dexmedetomidine and Remifentanil Continuous Infusion in Videolaparoscopic Cholecystectomy. Comparative Study

BACKGROUND AND OBJECTIVES: Dexmedetomidine has been used for sedation and as coadjuvant drug in general anesthesia. This study aimed at evaluating cardiovascular and sympathetic-adrenal responses to tracheal intubation and pneumoperitoneum inflation with dexmedetomidine, as compared to remifentanil during anesthesia with sevoflurane for videolaparoscopic cholecystectomy.

METHODS: Forty two physical status ASA I or II patients, aged 25 to 55 years, were randomly distributed in two groups: GI and GII. Anesthesia was induced with $1 \mu \mathrm{g} . \mathrm{kg}^{-1}$ dexmedetomidine (GI) or remifentanil (GII) continuous infusion for 10 minutes, followed by propofol and cisatracurium. Anesthesia was maintained with $0.7 \mu \mathrm{g} \cdot \mathrm{kg}^{-1} \cdot \mathrm{h}^{-1}$ dexmedetomidine or $0.5 \mu \mathrm{g} \cdot \mathrm{kg}^{-1} \cdot \mathrm{h}^{-1}$ remifentanil continuous infusion and different sevoflurane concentrations. SBP, DBP and HR were recorded in the following moments: $M_{1}$ before initial drug infusion; $M_{2}$ - after end of initial drug infusion; $M_{3}$ - after tracheal intubation; $M_{4}$ - before pneumoperitoneum; $M_{5}$ - after pneumoperitoneum; $M_{6}$ - five minutes after pneumoperitoneum deflation; $M_{7}$ - after tracheal extubation. Epinephrine and norepinephrine were dosed in $M_{4}, M_{5}$ and $M_{6}$. Sevoflurane expired concentration (EC), EC/MAC ratio and sevoflurane consumption were recorded in $M_{4}, M_{5}$ and $M_{6}$.

RESULTS: SBP and DBP variations were higher in the dexmedetomidine group in $M_{4}$ and $M_{5}$. HR, epinephrine and norepinephrine levels were similar between groups. Sevoflurane $E C$ was higher in $M_{4}$ and $M_{6}$ for $\mathrm{Gl}$, as well as EC/MAC ratio. There has been a higher sevoflurane consumption in $\mathrm{Gl}$, with a trend to less analgesics and anti-emetics consumption.

CONCLUSIONS: In the conditions of our study, dexmedetomidine has inhibited catecholamine release during tracheal intubation and pneumoperitoneum, however, has not prevented blood pressure increase in response to peritoneal inflation.

Key Words: ANALGESICS, Opioids: remifentanil; HIPNOTICS: dexmedetomidine; SURGERY, Videolaparoscopic: cholecystectomy 


\section{INTRODUÇÃO}

$V_{t}$ ários estudos demonstram as vantagens da colecistectomia videolaparoscópica como redução da dor pós-operatória, melhoria da função pulmonar e menor tempo de permanência hospitalar. Entretanto, a insuflação de $\mathrm{CO}_{2}$ para obter pneumoperitônio induz a ativação de mecanismos neuroendócrinos, desencadeando alterações cardiovasculares e hormonais. Algumas evidências sugerem que a técnica anestésica pode diminuir esta resposta, reduzindo a morbimortalidade do procedimento ${ }^{1,2}$

Os opióides são analgésicos potentes cuja utilização em altas doses implicam na redução da resposta neuroendócrina ao estresse cirúrgico ${ }^{3}$, induzem aumento no tempo de despertar e elevam a incidência de náuseas e vômitos no pós-operatório ${ }^{4,5}$. O remifentanil é um opióide sintético com efeitos farmacológicos similares aos dos potentes agonistas $\mu$ com meia-vida de ação curta e capacidade de promover supressão efetiva da resposta endócrina induzida pelo estresse cirúrgico ${ }^{6}$.

Nos últimos anos, estudos utilizando $\alpha_{2}$-agonistas demonstraram efeitos benéficos dessas drogas em bloquear a resposta neuroendócrina ao trauma operatório ${ }^{7-9}$. A clonidina atenua a resposta hemodinâmica à laringoscopia e à intubação traqueal ${ }^{9,10}$, também reduz as necessidades de anestésicos inalatórios durante estimulação cirúrgica ${ }^{11,12}$. A dexmedetomidina é uma droga agonista do receptor $\alpha_{2}$ com alta seletividade, que atua reduzindo o tônus simpático, diminuindo a pressão arterial e a freqüência cardíaca ${ }^{13,14}$; além disso, possui propriedades sedativas que levaram à sua utilização em terapia intensiva ${ }^{15}$ e atualmente como um adjuvante na anestesia ${ }^{16-18}$. Como medicação pré-anestésica, a dexmedetomidina reduz a resposta simpático-adrenal e cardiovascular à laringoscopia e à intubação orotraqueal, assim como o consumo de opióides no per e pós-operatório ${ }^{19}$.

No presente estudo avaliamos a utilização da dexmedetomidina em infusão contínua em anestesia geral com sevoflurano, traçando o perfil da resposta cardiovascular e metabólica ao estresse cirúrgico, comparando-a ao remifentanil. Estudamos ainda a influência das duas drogas na resposta simpático-adrenal à insuflação do pneumoperitônio através da dosagem de catecolaminas plasmáticas, o consumo de sevoflurano, o potencial analgésico e a incidência de náusease vômitos no pós-operatório imediato.

\section{MÉTODO}

Após aprovação pela Comissão de Ética da Faculdade de Medicina da Universidade Federal do Ceará, foram selecionados 42 pacientes estado físico ASA I e II, com idades entre 25 e 55 anos, de ambos os sexos, sem história de cardiopatia, nefropatia e/ou hepatopatia. Os pacientes foram aleatoriamente distribuídos, por meio de sorteio, em dois grupos. $\mathrm{O}$ grupo I recebeu dexmedetomidina, e o grupo II recebeu remifentanil. O estudo foi prospectivo e duplamente encoberto, caráter este garantido pela presença de um responsável pela preparação das drogas e manipulação da infusão, e de um anestesiologista encarregado do procedimento anestésico, anotação dos dados e avaliação pós-operatória. Todos os procedimentos cirúrgicos foram realizados pelo mesmo cirurgião.

Utilizou-se $200 \mu \mathrm{g}$ de dexmedetomidina diluída em $98 \mathrm{ml}$ de solução fisiológica a $0,9 \%$, obtendo-se uma concentração de $2 \mu \mathrm{g} \cdot \mathrm{ml}^{-1}$ no grupo I, enquanto no grupo Il empregou-se 2 $\mathrm{mg}$ de remifentanil diluído em $110 \mathrm{ml}$ de solução fisiológica a $0,9 \%$, com concentração de $18 \mu \mathrm{g} \cdot \mathrm{ml}^{-1}$. Todos os pacientes foram hidratados com solução de Ringer com lactato no ritmo de infusão de $4 \mathrm{ml} \cdot \mathrm{kg}^{-1} \cdot \mathrm{h}^{-1}$.

Os pacientes foram avaliados no pré-operatório e monitorizados durante o procedimento cirúrgico com ECG contínuo em duas derivações $D_{\|}$e $V 5$, oxímetro de pulso, pressão arterial não invasiva, capnografia e analisador de gases.

Aindução da anestesia teve início com infusão venosa contínua de dexmedetomidina ou remifentanil, ambas na dosagem de $1 \mu \mathrm{g} . \mathrm{kg}^{-1}$ durante 10 minutos ${ }^{20}$, juntamente com oxigênio a $100 \%$ sob máscara. A infusão foi precedida pela administração venosa de $0,02 \mathrm{mg} \cdot \mathrm{kg}^{-1}$ de atropina. Após o término da infusão inicial, seguiu-se, imediatamente, a infusão de manutenção nas doses de $0,7 \mu \mathrm{g} \cdot \mathrm{kg}^{-1} \cdot \mathrm{h}^{-1}$ de dexmedetomidina ou $0,5 \mu \mathrm{g} \cdot \mathrm{kg}^{-1} \cdot \mathrm{h}^{-1}$ de remifentanil. A hipnose foi obtida com 2,5 mg. $\mathrm{kg}^{-1}$ de propofol. Administrou-se 0,2 mg. $\mathrm{kg}^{-1} \mathrm{de}$ cisatracúrio para facilitação da intubação traqueal (IT). Após IT o paciente foi posicionado com o dorso elevado a aproximadamente $45^{\circ}$ e ligeiramente lateralizado para esquerda.

A infusão contínua de dexmedetomidina ou remifentanil foi mantida em cada grupo sem alteração da dose durante todo o procedimento operatório associado ao sevoflurano com concentração inicial de $2 \%$ e ajustes da concentração expirada para manter os parâmetros hemodinâmicos sem variações maiores que $30 \%$ dos valores basais.

Foram avaliadas a freqüência cardíaca (FC), pressão arterial sistólica (PAS) e pressão arterial diastólica (PAD) nos seguintes momentos: $\mathrm{M}_{1}$ - após monitorização, antes do início da infusão inicial da droga em estudo; $\mathrm{M}_{2}$-após término da infusão inicial da droga, antes da indução da anestesia; $M_{3}$ imediatamente após intubação traqueal; $\mathrm{M}_{4}$ - imediatamente antes do início do pneumoperitônio; $M_{5}$ - após estabelecimento do pneumoperitônio (pressão de insuflação de 10 $\mathrm{mmHg}$ ); $\mathrm{M}_{6}$ - cinco minutos após desinsuflado o pneumoperitônio e $\mathrm{M}_{7}$ - imediatamente após a extubação traqueal.

A concentração expirada (CE) do sevoflurano foi registrada por analisador de gases e anotada em todos os tempos de avaliação. No final do procedimento, o consumo total de sevoflurano (em $\mathrm{ml})$ foi registrado.

Três amostras venosas foram coletadas para avaliar os níveis sangüíneos de adrenalina e noradrenalina nos seguintes momentos: $M_{4}, M_{5}$ e $M_{6}$. O sangue foi coletado em frascos com EDTA, protegidos da luz e armazenados em refrigerador a $-20^{\circ} \mathrm{C}$ até o final da cirurgia. As amostras passaram por processo de centrifugação, sendo o plasma estocado em câmara fria com temperatura de $-70^{\circ} \mathrm{C}$. O método utilizado para dosagem sangüínea da adrenalina e noradrenalina plasmática foi a cromatografia líquida de alta resolução com níveis Vol. 53, N 4, Julho - Agosto, 2003 
avaliados em picogramas $/ \mathrm{ml}\left(\mathrm{pg} . \mathrm{ml}^{-1}\right)$. Os valores de referência foram para adrenalina $<150 \mathrm{pg} \cdot \mathrm{ml}^{-1}$ e noradrenalina $<$ $370 \mathrm{pg} \cdot \mathrm{ml}^{-1} 21$.

O tempo de despertar, considerado desde a interrupção do sevoflurano até a retirada do tubo traqueal, foi registrado, assim como a incidência de náuseas, vômitos e o tempo necessário para a utilização de analgésicos nas primeiras seis horas de pós-operatório quando da ocorrência de dor referida verbalmente pelo paciente.

Os parâmetros hemodinâmicos (PAS, PAD e FC), a relação CE/CAM, as concentrações plasmáticas de adrenalina e noradrenalina foram analisados pelo teste de Tukey, utilizando o nível de significância de $5 \%$ de forma que os experimentos foram realizados em parcelas subdivididas e testados nos dois tratamentos principais (dexmedetomidina e remifentanil) segundo o tempo. Para avaliação do tempo anestésico, tempo de insuflação do pneumoperitônio, consumo de sevoflurano, foi usado o teste $t$ Student nos sete tempos de avaliação. A incidência de dor no pós-operatório foi avaliada pelo teste Log-Rank. Os dados foram apresentados como média \pm desvio padrão.

Valores de $p<0,05$ foram considerados estatisticamente significantes.

\section{RESULTADOS}

Os dados demográficos das amostras estudadas estão apresentados na tabela l e foram considerados homogêneos. As variações na PAS e na PAD foram significativamente maiores nos momentos $\mathrm{M}_{4}$ e $\mathrm{M}_{5}$ no grupo I (dexmedetomidina) quando comparada ao grupo II (remifentanil) $(p<0,05)$ (Tabela II, Figuras 1 e 2). AFC e os níveis de adrenalina e noradrenalina não apresentaram diferença significativa entre os grupos I e II durante todo o procedimento (Tabelas II e III, Figuras 3, 4 e 5). ACE e a relação CE/CAM do sevoflurano foi significativamente maior nos momentos $M_{4}$ e $M_{6}$ do grupo I (dexmedetomidina) $(p<0,05)$, assim como o consumo do agente inalatório foi significativamente maior no grupo I ao longo do estudo $(p<0,05)$ (Tabelas IVe V, Figuras 6, 7 e 8).

Tabela I - Dados Demográficos

\begin{tabular}{lcc}
\hline Parâmetros & Grupo I & Grupo II \\
\hline Idade (anos) ${ }^{*}$ & $41,76 \pm 12,08$ & $42,70 \pm 9,24$ \\
Peso $(\mathrm{kg})^{*}$ & $71,14 \pm 10,38$ & $72,25 \pm 18,22$ \\
Altura $(\mathrm{cm})^{*}$ & $1,66 \pm 0,04$ & $1,67 \pm 0,08$ \\
Sexo & & \\
$\quad$ Masculino & 4 & 7 \\
$\quad$ Feminino & 17 & 14 \\
\hline
\end{tabular}

* Valores expressos pela Média \pm DP

A incidência de dor no pós-operatório foi estatisticamente menor no GI (Tabela VI e Figura 9). Quatro pacientes do GI $(28 \%)$ e oito do GII (42\%) utilizaram antiemético nas seis horas iniciais do pós-operatório.

Revista Brasileira de Anestesiologia

Vol. 53, № 4, Julho - Agosto, 2003

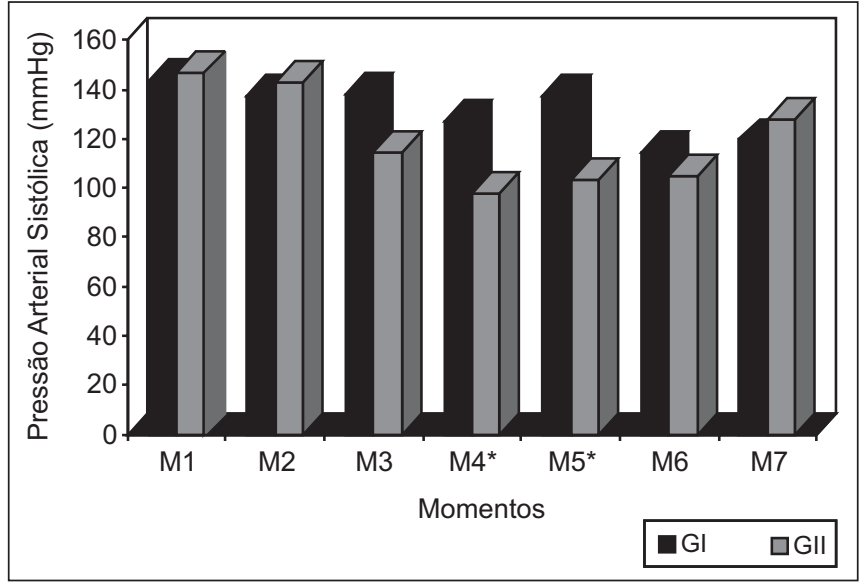

Figura 1 - Média de Pressão Arterial Sistólica (PAS); PAS em $\mathrm{M}_{4}$ e $\mathrm{M}_{5}$ do $\mathrm{GI}>\mathrm{Gll}$ * $(p<0,05)$

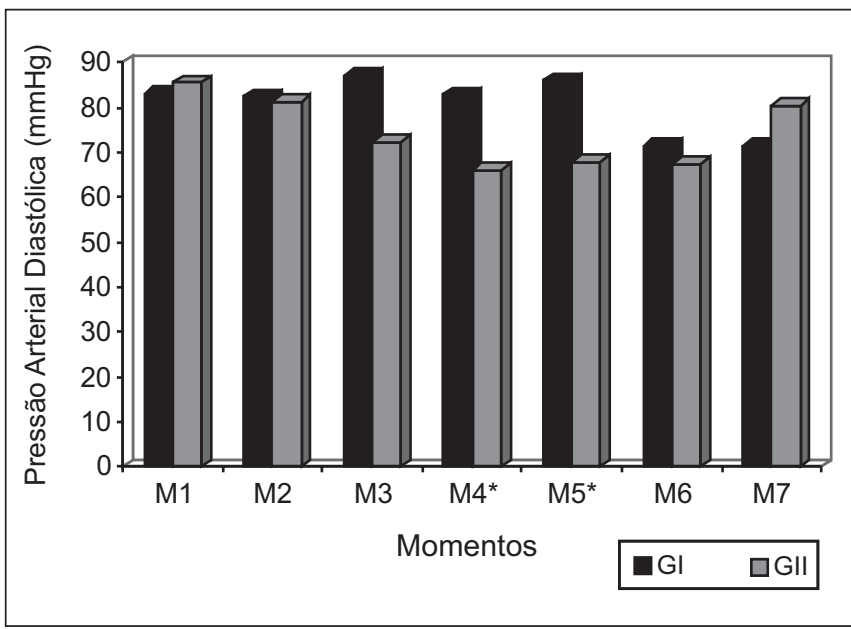

Figura 2 - Média de Pressão Arterial Diastólica (PAD); $\mathrm{PAD}$ em $\mathrm{M}_{4}$ e $\mathrm{M}_{5}$ do $\mathrm{GI}>\mathrm{GII}$ * $(p<0,05)$

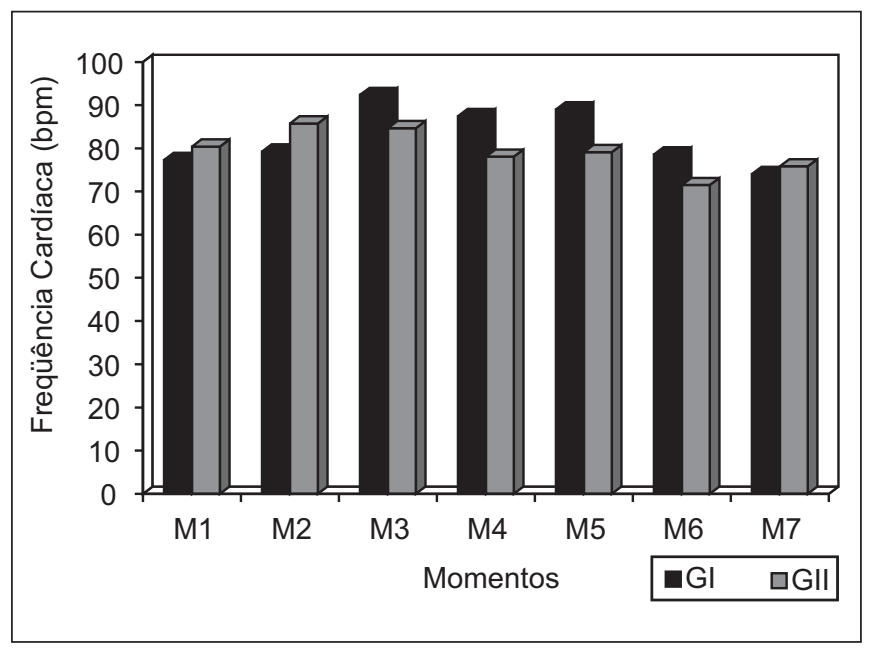

Figura 3 - Média de Freqüência Cardíaca (FC); FC não há diferença estatística entre GI e GII $(p>0,05)$ 
Tabela II - Pressão Arterial Sistólica (PAS), Pressão Arterial Diastólica (PAD), Freqüência Cardíaca (FC) (Média \pm DP)

\begin{tabular}{lcccccc} 
Momentos & \multicolumn{2}{c}{ PAS } & \multicolumn{2}{c}{ PAD } & GC \\
\hline$M_{1}$ & GI & GII & GI & GII & GI \\
$M_{2}$ & $144,05 \pm 21,78$ & $146,76 \pm 24,71$ & $83,05 \pm 12,09$ & $85,43 \pm 12,49$ & $77,33 \pm 15,29$ & $80,43 \pm 14,89$ \\
$M_{3}$ & $137,09 \pm 21,02$ & $143,00 \pm 42,02$ & $82,29 \pm 14,82$ & $81,29 \pm 12,45$ & $79,29 \pm 14,17$ & $85,76 \pm 16,13$ \\
$M_{4}$ & $138,00 \pm 21,93$ & $114,52 \pm 35,56$ & $87,14 \pm 16,89$ & $72,29 \pm 24,90$ & $92,38 \pm 24,67$ & $84,67 \pm 11,88$ \\
$M_{5}$ & $127,29 \pm 18,40^{*}$ & $97,81 \pm 19,31$ & $82,81 \pm 11,82^{*}$ & $66,00 \pm 13,22$ & $87,45 \pm 15,58$ & $78,09 \pm 8,13$ \\
$M_{6}$ & $137,67 \pm 19,70^{*}$ & $103,62 \pm 23,49$ & $85,86 \pm 17,39^{*}$ & $67,71 \pm 18,42$ & $89,00 \pm 18,50$ & $79,14 \pm 10,95$ \\
$M_{7}$ & $114,19 \pm 12,59$ & $105,24 \pm 19,90$ & $71,29 \pm 13,44$ & $67,38 \pm 17,26$ & $78,62 \pm 12,09$ & $71,52 \pm 12,92$ \\
\hline
\end{tabular}

* = Nível de significância $p<0,05$

Tabela III - Adrenalina e Noradrenalina Plasmática (pg. $\mathrm{ml}^{-1}$ ) (Média \pm DP)

\begin{tabular}{lcccc}
\hline Momentos & \multicolumn{2}{c}{ Adrenalina } & Noradrenalina \\
\hline$M_{4}$ & GI & GII & GI & GII \\
$M_{5}$ & $39,71 \pm 22,61$ & $48,19 \pm 25,92$ & $206,66 \pm 128,99$ & $201,76 \pm 94,93$ \\
$M_{6}$ & $48,67 \pm 31,87$ & $43,86 \pm 26,71$ & $212,00 \pm 96,75$ & $210,19 \pm 120,66$ \\
\hline
\end{tabular}

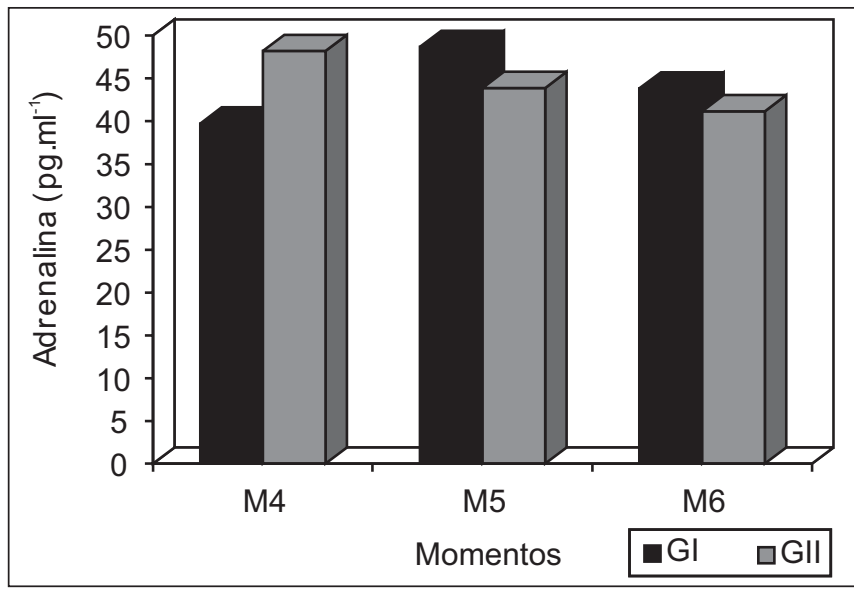

Figura 4 - Média dos Níveis de Adrenalina não Diferiu Significativamente entre GI e GII $(p>0,05)$

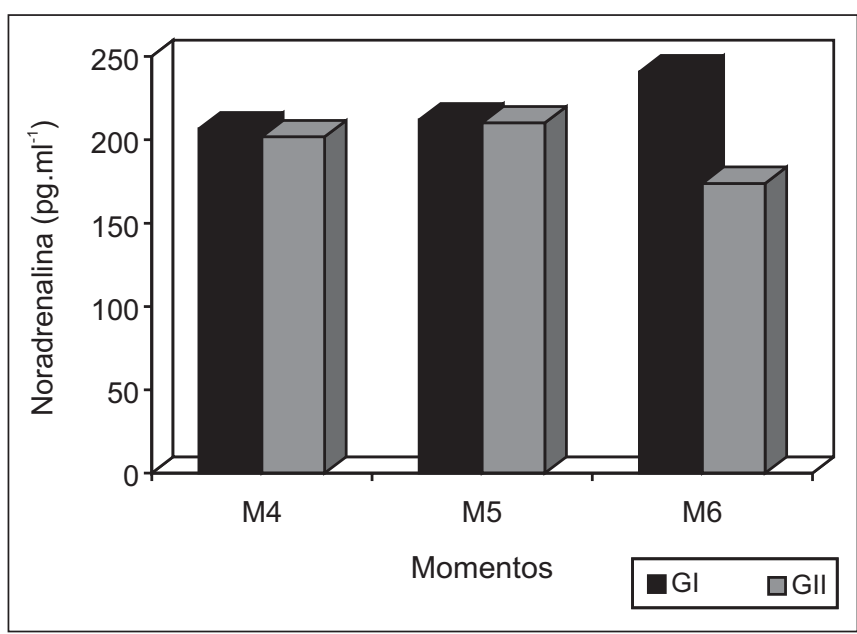

Figura 5 - Média dos Níveis de Noradrenalina não Diferiu Significativamente entre GI e GII $(p>0,05)$
Tabela IV - Concentração Expirada (CE\%) e Relação CE/CAM

\begin{tabular}{lcccc}
\hline Momentos & \multicolumn{2}{c}{ CE } & \multicolumn{2}{c}{ CE/CAM } \\
\hline$M_{4}$ & GI & GII & GI & GII \\
$M_{5}$ & $1,93 \pm 0,66^{*}$ & $1,73 \pm 0,81$ & $1,46 \pm 0,33^{*}$ & $0,87 \pm 0,41$ \\
$M_{6}$ & $1,69 \pm 0,33^{*}$ & $1,09 \pm 0,56$ & $0,84 \pm 0,17^{*}$ & $0,54 \pm 0,28$ \\
\hline
\end{tabular}

* $=$ Nível de significância $p<0,05$

Tabela V - Consumo de Sevoflurano durante o Experimento

\begin{tabular}{lcc}
\hline Momento & \multicolumn{2}{c}{ Sevoflurano $\left(\mathrm{ml}^{-\mathrm{min}^{-1}}\right)$} \\
\hline $\mathrm{M}_{4}$ & $\mathrm{Gl}$ & $\mathrm{Gll}$ \\
\hline
\end{tabular}

* $=$ Nível de significância $p<0,05$

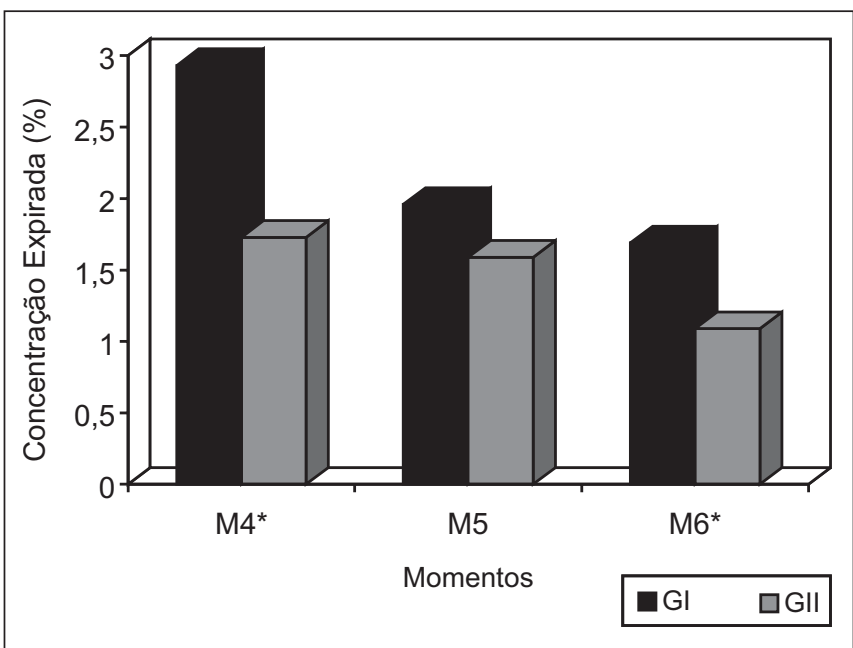

Figura 6 - Média de Concentração Expirada foi Significativamente Maior em $\mathrm{M}_{4}$ e $\mathrm{M}_{6}$ do $\mathrm{Gl}$ $*(p<0,05)$ 


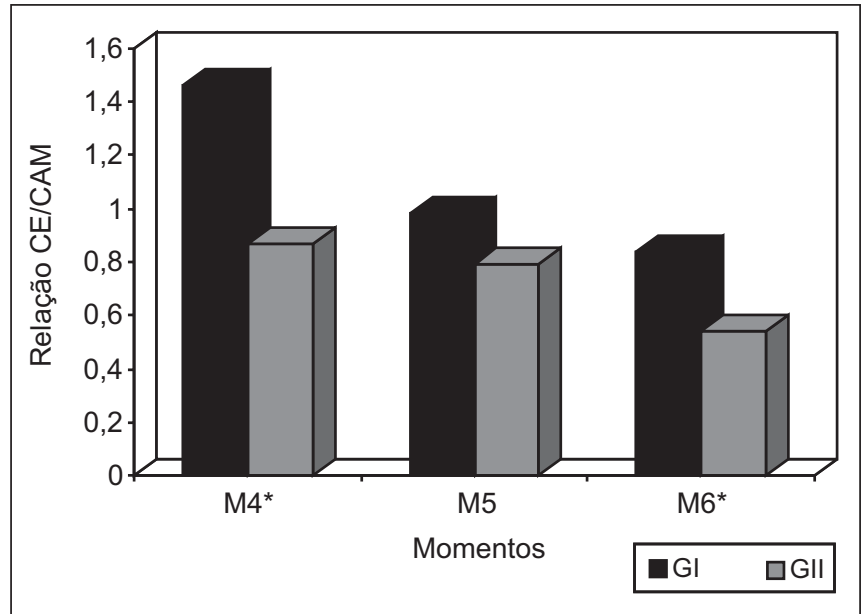

Figura 7 - Relação CE/CAM foi Significativamente Maior em $\mathrm{M}_{4} \mathrm{e}$ $\mathrm{M}_{6}$ do $\mathrm{Gl}$

* $(p<0,05)$

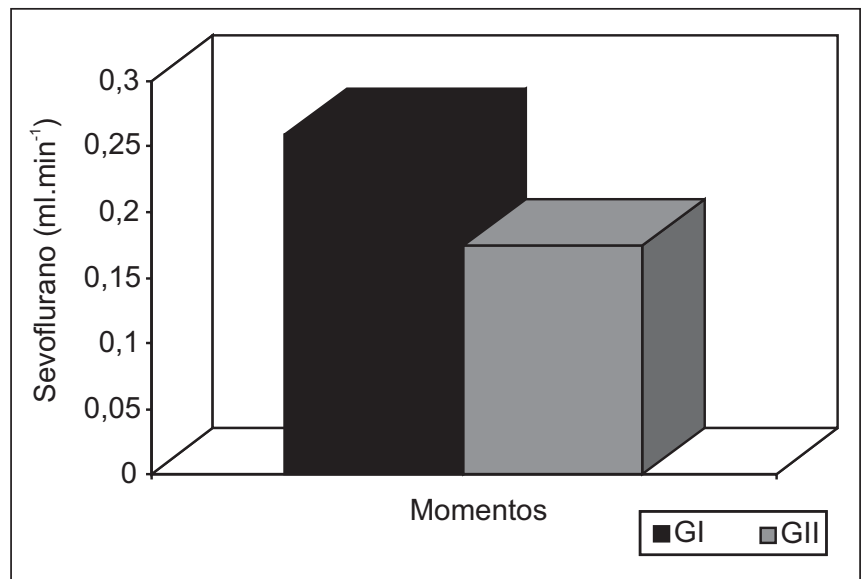

Figura 8 - Consumo de Sevoflurano foi Significativamente Maior no Gl

$(p<0,05)$

Tabela VI - Incidência de Dor no Pós-Operatório

\begin{tabular}{lccc}
\hline Grupos & $\begin{array}{c}\text { Tempo Decorrido entre } \\
\text { Anestesia e Dor (min) }\end{array}$ & $\begin{array}{c}\text { Ocorrência de Dor } \\
\left(\mathrm{N}^{\circ} \text { de Pacientes) }\right.\end{array}$ & $\begin{array}{c}\text { Densidade de } \\
\text { Incidência }\end{array}$ \\
\hline $\mathrm{GI}$ & 6335 & 7 & $0,00110^{*}$ \\
$\mathrm{GII}$ & 3658 & 12 & $0,00328^{*}$ \\
\hline Total & 9993 & 19 & 0,00190 \\
\hline
\end{tabular}

* = Nível de significância $p<0,05$

\section{DISCUSSÃO}

Oremifentanil é um opióide sintético que exibe efeitos farmacológicos dos agonistas de receptores opióides $\mu$. A dexmedetomidina é um $\alpha_{2}$-agonista com propriedades sedativas que reduz o tônus simpático, diminuindo a freqüência cardía-

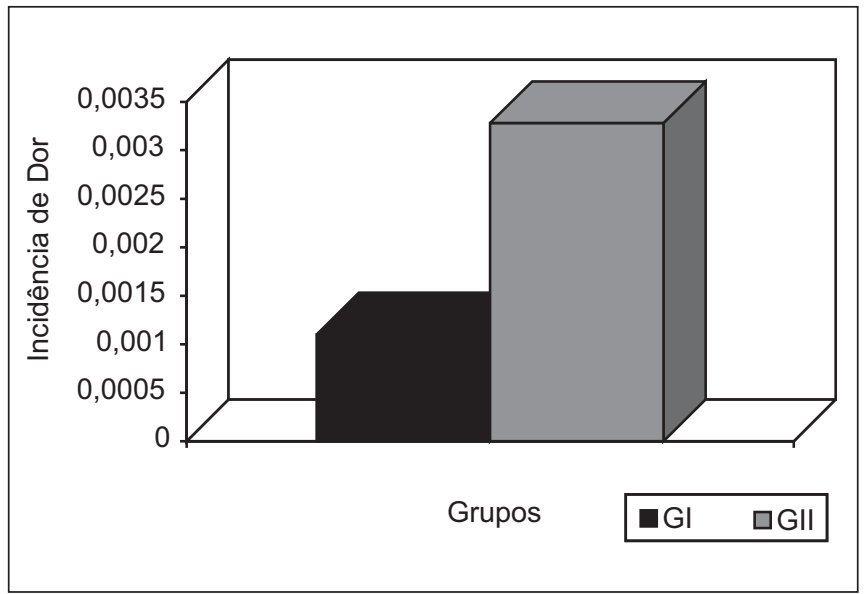

Figura 9 - Incidência de Dor no Pós-Operatório foi Significativamente Menor no GI

$(\mathrm{p}<0,05)$

ca e a pressão arterial. É utilizada em terapia intensiva e alguns estudos apontam sua eficácia como droga adjuvante em anestesia, reduzindo a CAM dos anestésicos inalatórios $16,17,22$, assim como as necessidades de tiopental e de propofol 18,20 .

Em estudos prévios, a administração de dexmedetomidina in bolus nas doses de $1 \mu \mathrm{g} . \mathrm{kg}^{-1}$ promoveu aumento transitório da pressão arterial e bradicardia reflexa, sendo portanto recomendado por alguns autores o uso rotineiro de anticolinérgicos associados aos $\alpha_{2}$-agonistas ${ }^{22}$. Outros autores, entretanto, defendem que a dexmedetomidina não deve ser utilizada in bolus, pois a resposta hipertensiva pode surgir devido a sua ligação ao receptor $\alpha_{2} b^{23}$. Nesse trabalho, a dose inicial de $1 \mu \mathrm{g} . \mathrm{kg}^{-1}$ de dexmedetomidina durante $10 \mathrm{mi}-$ nutos precedida pela atropina não induziu variação na FC, PAS e PAD. É importante ressaltar que a ausência de bradicardia reflexa com o uso da dexmedetomidina pode ser devida à dose de $0,02 \mathrm{mg} \cdot \mathrm{kg}^{-1}$ de atropina administrada na indução da anestesia. Desta forma, não verificamos elevação da pressão arterial ou bradicardia após infusão de dexmedetomidina em nenhum dos pacientes avaliados.

A PAS, PAD, FC, noradrenalina e adrenalina em resposta a intubação orotraqueal não apresentaram diferença significativa entre os grupos I e II, na dose utilizada. Tais resultados sugerem que a dexmedetomidina na dose utilizada, associada ao propofol $\left(2,5 \mathrm{mg} \cdot \mathrm{kg}^{-1}\right)$, bloqueou efetivamente a resposta cardiovascular decorrente da intubação traqueal de maneira similar ao remifentanil.

As alterações cardiovasculares na colecistectomia videolaparoscópica se manifestam basicamente pelo aumento na PAS, PAD e FC no per-operatório e durante o pneumoperitônio ${ }^{23}$. Em nosso estudo houve em $\mathrm{M}_{5}$ um aumento estatisticamente significante na PAS, PAD no grupo da dexmedetomidina sem, contudo, alterar a FC, assim sendo, a CE e a CE/CAM foram significativamente maior neste tempo, visando manter níveis pressóricos previamente estabelecidos no protocolo. 
Outros autores ${ }^{24}$ demonstraram que o remifentanil na dose de $8 \mu \mathrm{g} . \mathrm{kg}^{-1}$ é capaz de bloquear a liberação da vasopressina durante o pneumoperitônio em cirurgia videolaparoscópica, o que provavelmente mantém os níveis de PAS e PAD dentro de parâmetros aceitáveis para o procedimento. Os $\alpha_{2}$-agonistas em colecistectomia videolaparoscópica, mais precisamente a clonidina na dose de $8 \mu \mathrm{g} \cdot \mathrm{kg}^{-1}$ em infusão durante 1 (uma) hora, foi incapaz de bloquear efetivamente a liberação da vasopressina plasmática durante o pneumoperitônio. Esses dados vão a favor do presente estudo, em que observamos uma elevação da PAS e PAD no $\mathrm{M}_{4}$ e $\mathrm{M}_{5}$, provavelmente mediado por aumento da resistência vascular sistêmica. É provável que a dexmedetomidina, na dose utilizada, não bloqueie a liberação da vasopressina e do cortisol plasmático, mediando assim uma elevação na resistência vascular sistêmica, o que leva a aumento da PAS e PAD ${ }^{25}$.

O aumento do metabolismo celular constitui um componente importante da resposta ao estresse cirúrgico, podendo comprometer pacientes mais graves ou aqueles submetidos a procedimentos cirúrgicos de grande porte. Aumentos nas concentrações plasmáticas de catecolaminas elevam o risco cardíaco e o catabolismo protéico, retardando a recuperação. Outros estudos ${ }^{26}$ demonstraram que a laparoscopia resulta em progressivo e significante aumento nas concentrações plasmáticas de cortisol, adrenalina, noradrenalina, renina e vasopressina, enquanto que outros autores avaliando apenas o comportamento dos níveis plasmáticos de catecolaminas verificaram aumentos nos mesmos, relacionados ao pneumoperitônio ${ }^{27-29}$.

Estudos prévios demonstraram que os opióides são potentes inibidores do eixo hipófise-adrenal ${ }^{30}$, prevenindo aumento na concentração plasmática de cortisol, glicose, catecolaminas e aldosterona durante e após circulação extracorpórea ${ }^{31,32}$. Em voluntários sadios, administrando dexmedetomidina por via venosa, houve inibição da atividade do sistema nervoso autônomo simpático, avaliada pela redução dos níveis de noradrenalina no plasma ${ }^{32}$.

Nesse trabalho os resultados de adrenalina e noradrenalina plasmáticos revelam que não houve variações estatisticamente significantes na resposta à insuflação e à deflação do pneumoperitônio em ambos os grupos. Assim, os dados sugerem que tanto a dexmedetomidina como o remifentanil, nas doses utilizadas, foram igualmente eficientes em bloquear a liberação de noradrenalina e adrenalina desencadeada pela insuflação do pneumoperitônio.

Apesar da demonstração de que ambas as drogas são eficientes em bloquear a liberação de catecolaminas decorrentes da insuflação do pneumoperitônio, é preciso ressaltar que não existiu correlação direta com os níveis pressóricos durante o pneumoperitônio, provavelmente a elevação da PAS se deva à vasopressina e ao cortisol, como já mencionado. Ou seja, para manter níveis pressóricos adequados, durante $\mathrm{M}_{4}$, foi utilizado uma $\mathrm{CE}$ do sevoflurano maior no grupo da dexmedetomidina.

A ocorrência de náuseas e vômitos no pós-operatório foi da ordem de $42 \%$ no grupo Il e de $28 \%$ no grupo I, sugerindo que a utilização de dexmedetomidina pode contribuir para redu- ção desses sintomas no pós-operatório de cirurgias videolaparoscópicas.

O número de pacientes que necessitou de analgésicos nas primeiras seis horas de pós-operatório foi menor no Gl do que no GII, o que chama a atenção para o possível efeito analgésico da dexmedetomidina.

Concluindo, a dexmedetomidina e o remifentanil nas doses empregadas foram eficientes em bloquear a resposta hemodinâmica à intubação traqueal, o mesmo não ocorrendo durante o pneumoperitônio, por haver aumento na PAS e PAD do grupo da dexmedetomidina (GI), apesar dos níveis de adrenalina e noradrenalina não diferirem do grupo do remifentanil (GII).

\section{Hemodynamic and Metabolic Evaluation of Dexmedetomidine and Remifentanil Continuous Infusion in Videolaparoscopic Cholecystectomy. Comparative Study}

Thatiany Pereira Chaves, M.D., Josenília Maria Alves Gomes, TSA, M.D., Francisco Elano Carvalho Pereira, M.D., Sara Lúcia Cavalcante, TSA, M.D., Ilse M. Tigre de Arruda Leitão, M.D., Hipólito Sousa Monte, M.D., Rodrigo Dornfeld Escalante, M.D.

\section{INTRODUCTION}

Several studies have shown advantages of videolaparoscopic cholecistectomy, such as postoperative pain decrease, pulmonary function improvement and shorter hospital stay. However, $\mathrm{CO}_{2}$ inflation for pneumoperitoneum induces the activation of neuroendocrine mechanisms triggering cardiovascular and hormonal changes. Some evidences suggest that the anesthetic technique may decrease this response, thus decreasing morbidity-mortality ${ }^{1,2}$.

Opioids are potent analgesics and, in high doses, they decrease neuroendocrine response to surgical stress ${ }^{3}$, induce longer emergence time and increase the incidence of postoperative nausea and vomiting ${ }^{4,5}$. Remifentanil is a synthetic opioid with pharmacological effects similar to those of potent $\mu$ agonists, short-action half-life and the ability to promote effective suppression of surgical stress-induced endocrine response.

Recent studies with $\alpha_{2}$-agonists have shown beneficial effects of such drugs in blocking neuroendocrine response to surgical trauma ${ }^{7-9}$. Clonidine attenuates hemodynamic response to laryngoscopy and tracheal intubation ${ }^{9,10}$, in addition to decreasing inhalational anesthetics consumption during surgical stimulation ${ }^{11,12}$. Dexmedetomidine is a highly selective $\alpha_{2}$ receptor agonist which decreases sympathetic tone, reducing blood pressure and heart rate ${ }^{13,14}$. Moreover, its sedative properties render it useful in intensive care practice ${ }^{15}$, and currently as anesthetic adjuvant ${ }^{16-18}$. As 
preanesthetic medication, dexmedetomidine decreases sympathetic-adrenal and cardiovascular response to laryngoscopy and tracheal intubation, as well as peri and postoperative opioid consumption ${ }^{19}$.

Our study has evaluated the use of dexmedetomidine continuous infusion in general anesthesia with sevoflurane, plotting cardiovascular and metabolic responses to surgical stress as compared to remifentanil. It has also evaluated the influence of both drugs in sympathetic-adrenal response to pneumoperitoneum inflation through the dosage of plasma catecholamines, sevoflurane consumption, analgesic potential and the incidence of immediate postoperative period nausea and vomiting.

\section{METHODS}

After the Ethics Committee, Faculdade de Medicina, Universidade Federal, Ceará approval, 42 patients of both genders, physical status ASA I and II, aged 25 to 55 years, with no history of heart, kidney and/or liver disease were included in the study. Patients were randomly distributed in two groups: $\mathrm{GI}=$ dexmedetomidine and GII = remifentanil. The prospective double-blind design of this study was assured by the presence of a person in charge of preparing drugs and manipulating infusions and of an anesthesiologist in charge of the anesthetic procedure, data collection and postoperative evaluation. All surgical procedures were performed by the same surgeon.

Group I infusion solution was made of $200 \mu \mathrm{g}$ dexmedetomidine diluted in $98 \mathrm{ml}$ of $0.9 \%$ saline solution, in a concentration of $2 \mu \mathrm{g} \cdot \mathrm{ml}^{-1}$. Group II was given solution made of $2 \mathrm{mg}$ remifentanil diluted in $110 \mathrm{ml}$ of $0.9 \%$ saline solution, in a concentration of $18 \mu \mathrm{g} \cdot \mathrm{ml}^{-1}$. All patients were hydrated with lactated Ringer's at an infusion rate of $4 \mathrm{ml} \cdot \mathrm{kg}^{-1} \cdot \mathrm{h}^{-1}$.

Patients were preoperatively evaluated and monitored during surgery with continuous ECG in $D_{\|}$and V5 leads, pulse oximetry, non-invasive blood pressure, capnography and blood gases analyzer.

Anesthesia was induced with dexmedetomidine or remifentanil continuous infusion, both with the dosage of 1 $\mu \mathrm{g} . \mathrm{kg}^{-1}$ during 10 minutes ${ }^{20}$, together with $100 \%$ oxygen under mask. Infusions were preceded by intravenous 0.02 $\mathrm{mg} . \mathrm{kg}^{-1}$ atropine. Anesthesia was then maintained with 0.7 $\mu \mathrm{g} . \mathrm{kg}^{-1} \cdot \mathrm{h}^{-1}$ dexmedetomidine or $0.5 \mu \mathrm{g} . \mathrm{kg}^{-1} \cdot \mathrm{h}^{-1}$ remifentanil. Hypnosis was obtained with $2.5 \mathrm{mg} . \mathrm{kg}^{-1}$ propofol. Patients received $0.2 \mathrm{mg} \cdot \mathrm{kg}^{-1}$ cisatracurium to help tracheal intubation ( $\mathrm{TI})$. After TI, patients were positioned with the trunk elevated approximately $45^{\circ}$ and slightly lateralized to the left.

Continuous dexmedetomidine and remifentanil infusions were kept fixed in both groups throughout the procedure, associated to sevoflurane at an initial concentration of $2 \%$. Expired concentration adjustments were made to avoid hemodynamic parameters variations higher than $30 \%$ of baseline values.
Heart rate (HR), systolic blood pressure (SBP) and diastolic blood pressure (DBP) were evaluated in the following moments: $\mathrm{M}_{1}$ - after monitoring and before initial infusion of the studied drug; $M_{2}$ - after initial infusion completion and before anesthetic induction; $M_{3}$ - immediately after tracheal intubation; $\mathrm{M}_{4}$ - immediately before pneumoperitoneum; $\mathrm{M}_{5}$ after pneumoperitoneum (10 $\mathrm{mmHg}$ inflation pressure); $\mathrm{M}_{6}$ five minutes after pneumoperitoneum deflation; and $\mathrm{M}_{7}$ - immediately after tracheal extubation.

Sevoflurane expired concentration (EC) was recorded by a blood gases analyzer in all evaluation times. At surgery completion, total sevoflurane consumption (in $\mathrm{ml}$ ) was recorded. Three venous samples were collected to evaluate blood epinephrine and norepinephrine levels in moments $M_{4}, M_{5}$ and $M_{6}$. Blood was collected in EDTA-containing vials, protected from light and stored in refrigerator at $-20^{\circ} \mathrm{C}$ until surgery completion. Samples underwent centrifugation and plasma was stored in a cold chamber at $-70^{\circ} \mathrm{C}$. High-resolution liquid chromatography was used to dose plasma epinephrine and norepinephrine and levels, expressed in picograms $\left(\right.$ pg. $\left.\mathrm{ml}^{-1}\right)$. Reference values were: epinephrine $<150 \mathrm{pg} \cdot \mathrm{ml}^{-1}$ and norepinephrine $<370 \mathrm{pg} \cdot \mathrm{ml}^{-1}$.

Emergence time, considered from sevoflurane withdrawal to tracheal extubation, was recorded, as well as the incidence of nausea and vomiting and the time elapsed until first analgesic request during the first 6 postoperative hours.

Hemodynamic parameters (SBP, DBP and HR), EC/MAC ratio, plasma epinephrine and norepinephrine concentrations were analyzed by Tukey's test with significance level of $5 \%$. Experiments were performed in subdivided parts and tested in two major treatments (dexmedetomidine and remifentanil) according to time. Student's $t$ test was used to evaluate anesthetic time, pneumoperitoneum inflation time and sevoflurane consumption in the seven evaluation times. Log-Rank test was used to evaluate the incidence of postoperative pain. Data are presented in mean \pm standard deviation. $\mathrm{P}<0.05$ was considered statistically significant.

\section{RESULTS}

Demographics data are shown in table I and were considered homogeneous. SBP and DPB changes were significantly higher in moments $\mathrm{M}_{4}$ and $\mathrm{M}_{5}$ for $\mathrm{Gl}$ (dexmedetomidine) as compared to GII (remifentanil) $(p<0.05)$ (Table II, Figures 1 and 2). There were no significant differences in HR, epinephrine and norepinephrine levels between GI and GII throughout the procedure (Tables II and III, Figures 3, 4 and $5)$. Sevoflurane EC and EC/MAC ratio were significantly higher in moments $\mathrm{M}_{4}$ and $\mathrm{M}_{6}$ for $\mathrm{GI}$ (dexmedetomidine) $(\mathrm{p}<$ $0.05)$. Inhalational agent consumption was significantly higher in GI along the study $(p<0.05)$ (Tables IV and V, Figures 6, 7 and 8). 
Table I - Demographics Data

\begin{tabular}{lcc}
\hline Parameters & Group I & Group II \\
\hline Age $(\text { years })^{*}$ & $41.76 \pm 12.08$ & $42.70 \pm 9.24$ \\
Weight $(\mathrm{kg})^{*}$ & $71.14 \pm 10.38$ & $72.25 \pm 18.22$ \\
Height $(\mathrm{cm}){ }^{*}$ & $1.66 \pm 0.04$ & $1.67 \pm 0.08$ \\
Gender & & \\
$\quad$ Male & 4 & 7 \\
$\quad$ Female & 17 & 14 \\
\hline
\end{tabular}

*Values expressed in Mean \pm SD

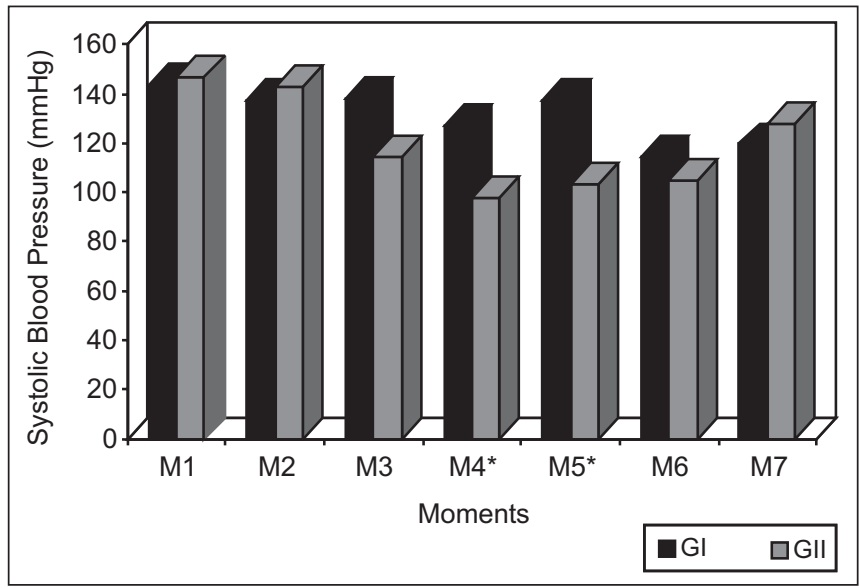

Figure 1 - Mean Systolic Blood Pressure (SBP);

$\mathrm{SBP}$ in $\mathrm{M}_{4}$ and $\mathrm{M}_{5}$ in $\mathrm{GI}>\mathrm{II}$

$(p<0.05)$

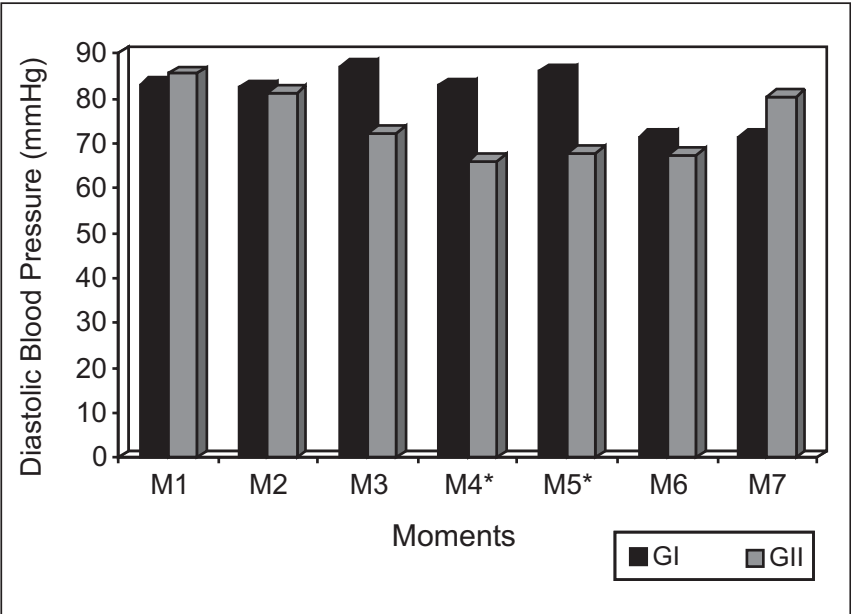

Figure 2 - Mean Diastolic Blood Pressure (DBP); $\mathrm{DBP}$ in $\mathrm{M}_{4}$ and $\mathrm{M}_{5}$ in $\mathrm{Gl}>\mathrm{II}$ $(p<0.05)$

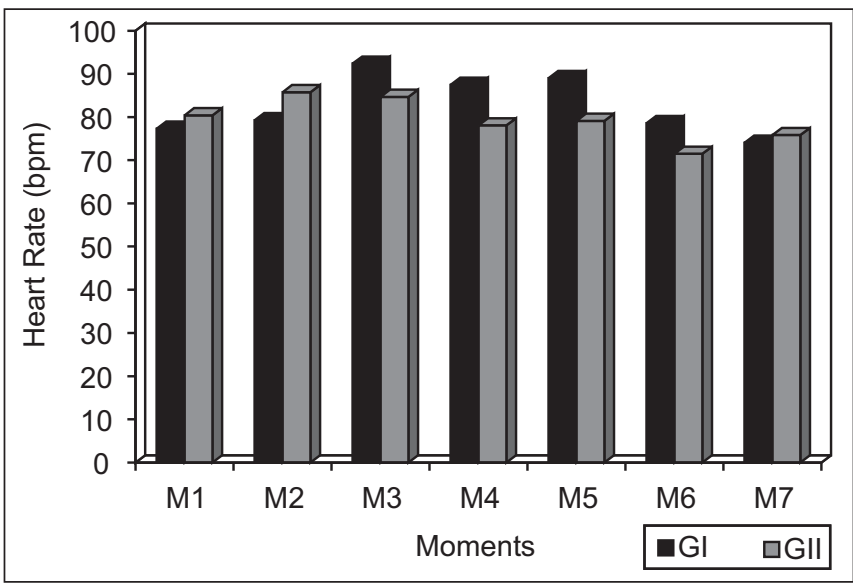

Figure 3 - Mean Heart Rate (HR).

no statistical difference in HR between $\mathrm{GI}$ and II $(p>0.05)$

Table II - Systolic Blood Pressure (SBP), Diastolic Blood Pressure (DBP), Heart Rate (HR) (Mean \pm SD)

\begin{tabular}{lcccccc}
\hline Moments & \multicolumn{2}{c}{ SBP } & \multicolumn{2}{c}{ DBP } & HR \\
\hline $\mathrm{M}_{1}$ & $\mathrm{Gl}$ & $\mathrm{I}$ & $\mathrm{Gl}$ & $\mathrm{II}$ & $\mathrm{Gl}$ \\
$\mathrm{M}_{2}$ & $144.05 \pm 21.78$ & $146.76 \pm 24.71$ & $83.05 \pm 12.09$ & $85.43 \pm 12.49$ & $77.33 \pm 15.29$ & $80.43 \pm 14.89$ \\
$\mathrm{M}_{3}$ & $137.09 \pm 21.02$ & $143.00 \pm 42.02$ & $82.29 \pm 14.82$ & $81.29 \pm 12.45$ & $79.29 \pm 14.17$ & $85.76 \pm 16.13$ \\
$\mathrm{M}_{4}$ & $138.00 \pm 21.93$ & $114.52 \pm 35.56$ & $87.14 \pm 16.89$ & $72.29 \pm 24.90$ & $92.38 \pm 24.67$ & $84.67 \pm 11.88$ \\
$\mathrm{M}_{5}$ & $127.29 \pm 18.40^{*}$ & $97.81 \pm 19.31$ & $82.81 \pm 11.82^{*}$ & $66.00 \pm 13.22$ & $87.45 \pm 15.58$ & $78.09 \pm 8.13$ \\
$\mathrm{M}_{6}$ & $137.67 \pm 19.70^{*}$ & $103.62 \pm 23.49$ & $85.86 \pm 17.39^{*}$ & $67.71 \pm 18.42$ & $89.00 \pm 18.50$ & $79.14 \pm 10.95$ \\
$\mathrm{M}_{7}$ & $114.19 \pm 12.59$ & $105.24 \pm 19.90$ & $71.29 \pm 13.44$ & $67.38 \pm 17.26$ & $78.62 \pm 12.09$ & $71.52 \pm 12.92$ \\
\hline
\end{tabular}

* $=$ Significance level $p<0.05$

Table III - Plasma Epinephrine and Norepinephrine $\left(\right.$ pg. $\left.\mathrm{ml}^{-1}\right)($ Mean \pm SD $)$

\begin{tabular}{lcccc}
\hline Moments & \multicolumn{2}{c}{ Epinephrine } & \multicolumn{2}{c}{ Norepinephrine } \\
\hline & $\mathrm{Gl}$ & $\mathrm{II}$ & $\mathrm{Gl}$ & $\mathrm{I}$ \\
\hline $\mathrm{M}_{4}$ & $39.71 \pm 22.61$ & $48.19 \pm 25.92$ & $206.66 \pm 128.99$ & $201.76 \pm 94.93$ \\
$\mathrm{M}_{5}$ & $48.67 \pm 31.87$ & $43.86 \pm 26.71$ & $212.00 \pm 96.75$ & $210.19 \pm 120.66$ \\
$\mathrm{M}_{6}$ & $43.81 \pm 29.63$ & $41.14 \pm 26.28$ & $240.76 \pm 137.05$ & $173.90 \pm 100.23$ \\
\hline
\end{tabular}


HEMODYNAMIC AND METABOLIC EVALUATION OF DEXMEDETOMIDINE AND REMIFENTANIL

CONTINUOUS INFUSION IN VIDEOLAPAROSCOPIC CHOLECYSTECTOMY. COMPARATIVE STUDY

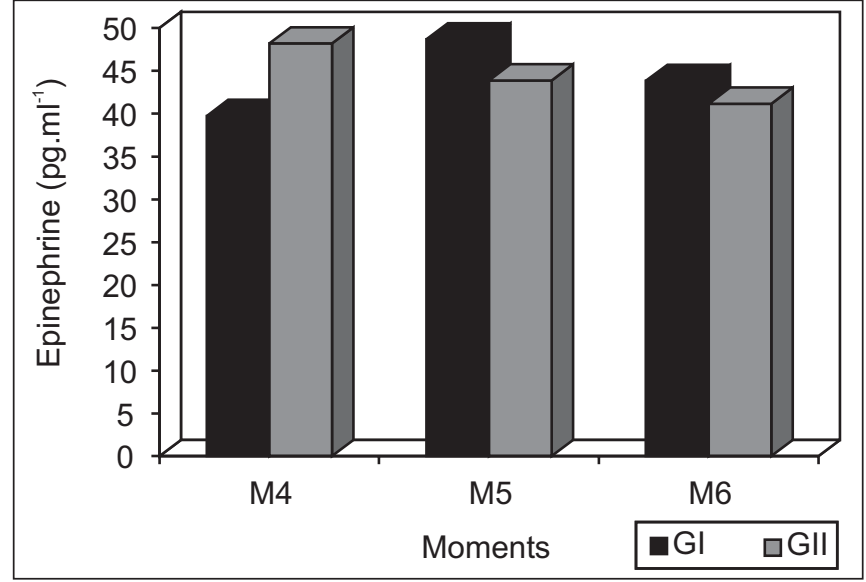

Figure 4 - Mean Epinephrine Levels were not Significantly Different between $\mathrm{Gl}$ and II $(p>0.05)$

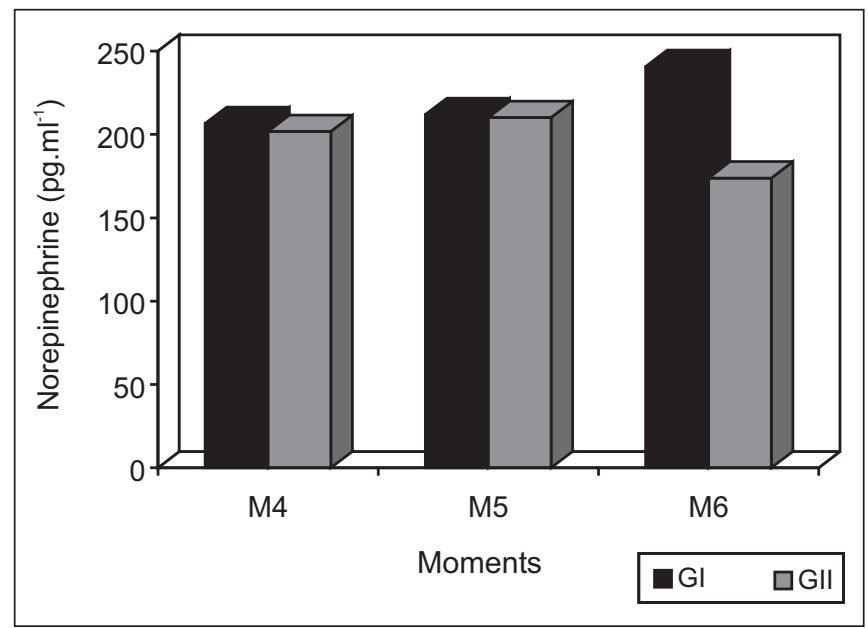

Figure 5 - Mean Norepinephrine Levels were not Significantly Different between $\mathrm{Gl}$ and II

$(p>0.05)$

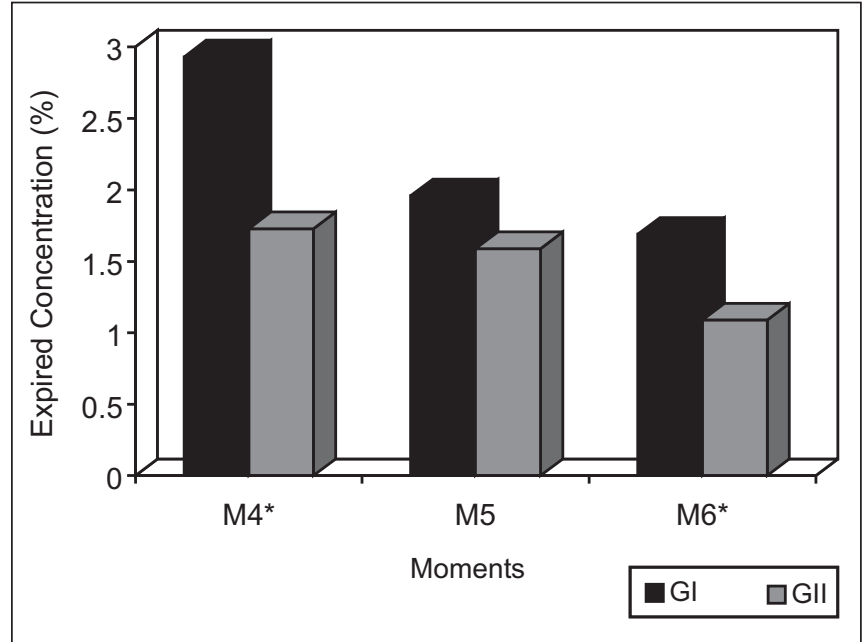

Figure 6 - Expired concentration mean was significantly higher in $\mathrm{M}_{4}$ and $\mathrm{M}_{6}$ for $\mathrm{Gl}$ $(p<0.05)$

Revista Brasileira de Anestesiologia Vol. 53, № 4, Julho - Agosto, 2003

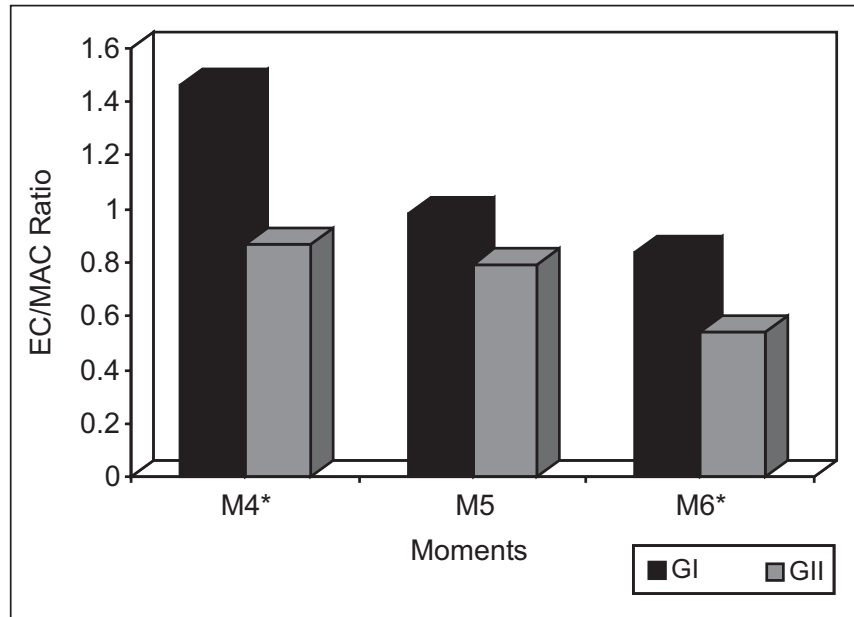

Figure 7 - EC/MAC ratio was significantly higher in $M_{4}$ and $M_{6}$ for $G I$ $(p<0.05)$

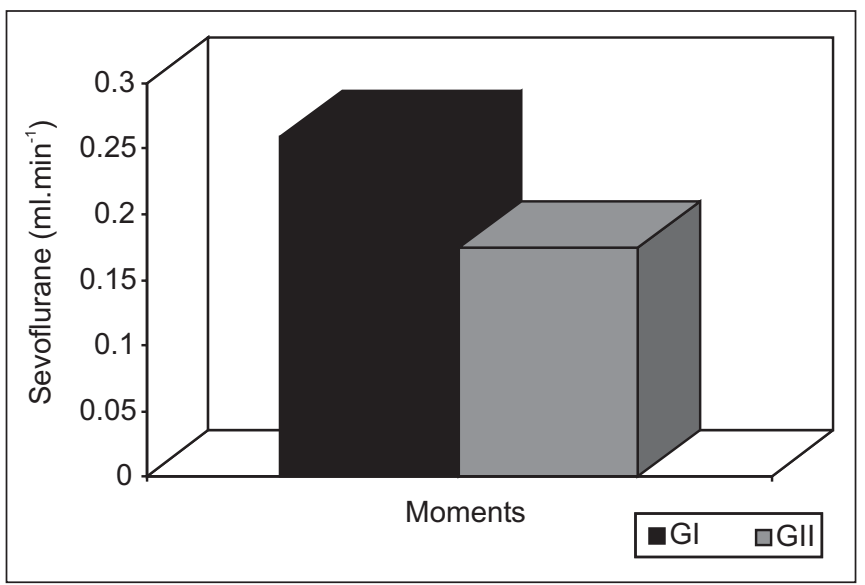

Figure 8 - Sevoflurane Consumption was significantly higher in GI $(p<0.05)$

Table IV - Expired Concentration (EC\%) and EC/MAC ratio

\begin{tabular}{lcccc}
\hline Moments & \multicolumn{2}{c}{ EC } & \multicolumn{2}{c}{ EC/MAC } \\
\hline & $\mathrm{GI}$ & $\mathrm{G} I \mathrm{G}$ & $\mathrm{Gl}$ & $\mathrm{G} I$ \\
\hline $\mathrm{M}_{4}$ & $2.93 \pm 0.66^{*}$ & $1.73 \pm 0.81$ & $1.46 \pm 0.33^{*}$ & $0.87 \pm 0.41$ \\
$\mathrm{M}_{5}$ & $1.96 \pm 0.40$ & $1.59 \pm 0.62$ & $0.98 \pm 0.20$ & $0.79 \pm 0.31$ \\
$\mathrm{M}_{6}$ & $1.69 \pm 0.33^{*}$ & $1.09 \pm 0.56$ & $0.84 \pm 0.17^{*}$ & $0.54 \pm 0.28$ \\
\hline
\end{tabular}

* $=$ Significance level $p<0.05$

Table V - Sevoflurane Consumption during the Experiment

\begin{tabular}{lcc}
\hline Moment & \multicolumn{2}{c}{ Sevoflurane $\left(\mathrm{ml}^{\mathrm{min}}{ }^{-1}\right)$} \\
\hline $\mathrm{M}_{4}$ & $\mathrm{Gl}$ & $\mathrm{GII}$ \\
\hline
\end{tabular}

${ }^{*}=$ Significance level $p<0.05$ 
The incidence of postoperative pain was significantly lower in GI (Table VI and Figure 9). Four GI patients (28\%) and eight II patients $(42 \%)$ needed anti-emetics in the first 6 postoperative hours.

\section{Table VI - Incidence of Postoperative Pain}

\begin{tabular}{lccc}
\hline Groups & $\begin{array}{c}\text { Time between Anesthesia } \\
\text { and Pain (min) }\end{array}$ & $\begin{array}{c}\text { Incidence of Pain } \\
\text { (N. of patients) }\end{array}$ & $\begin{array}{c}\text { Incidence } \\
\text { Density }\end{array}$ \\
\hline GI & 6335 & 7 & $0.00110^{*}$ \\
GII & 3658 & 12 & $0.00328^{*}$ \\
\hline Total & 9993 & 19 & 0.00190 \\
\hline
\end{tabular}

* = significance level $<0.05$

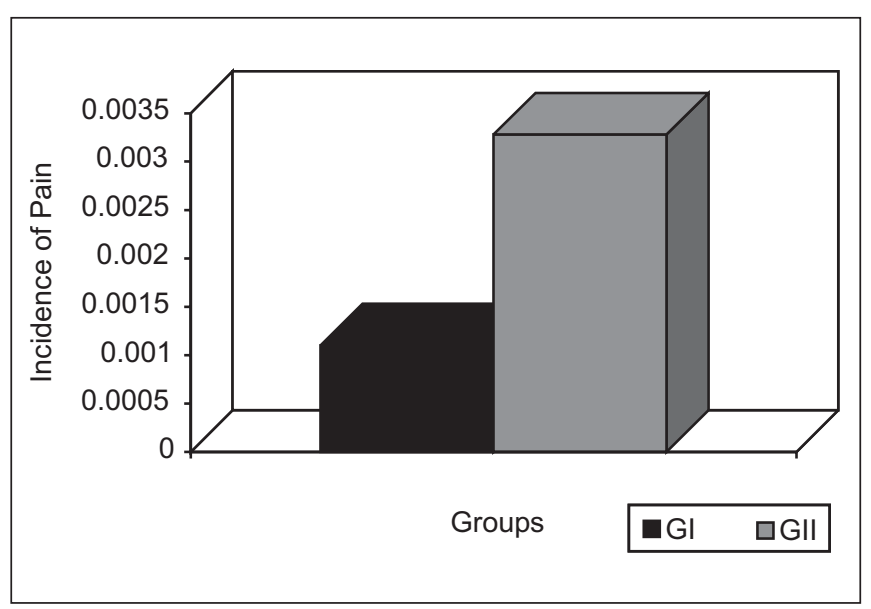

Figure 9 - Incidence of Postoperative Pain was Lower in GI $(p<0.05)$

\section{DISCUSSION}

Remifentanil is a synthetic opioid which shares the pharmacological effects of $\mu$ opioid receptor agonists. Dexmedetomidine is an $\alpha_{2}$-agonist with sedative properties which decreases sympathetic tone, heart rate and blood pressure. It is used in intensive care and some studies have shown ts efficacy as a coadjuvant drug in anesthesia, decreasing inhalational anesthetics MAC ${ }^{16,17,22}$, as well as thiopental and propofol requirements ${ }^{18,20}$.

In previous studies, bolus $1 \mu \mathrm{g} \cdot \mathrm{kg}^{-1}$ dexmedetomidine has promoted transient blood pressure increase and reflex bradycardia, thus the routine use of anticholinergics associated to $\alpha_{2}$-agonists has been recommended by some authors ${ }^{22}$. Others, however, advocate that dexmedetomidine should not be used in bolus since there might be hypertensive response due to its binding to $\alpha_{2}$ b receptor ${ }^{23}$. In our study, the initial $1 \mu \mathrm{g} . \mathrm{kg}^{-1}$ dexmedetomidine dose for 10 minutes preceded by atropine has not induced HR, SBP and DPB changes. It is important to stress that the absence of reflex bradycardia with dexmedetomidine might have been due to $0.02 \mathrm{mg} \cdot \mathrm{kg}^{-1}$ atropine administered during anesthetic induction. So, we have not observed blood pressure increase or bradycardia after dexmedetomidine in any evaluated patient.

SBP, DBP, HR, norepinephrine and epinephrine levels in response to tracheal intubation were not significantly different between groups I and II. Such results suggest that, similarly to remifentanil, dexmedetomidine in the dose used and associated to propofol $\left(2.5 \mathrm{mg} . \mathrm{kg}^{-1}\right)$ has effectively blocked cardiovascular response to tracheal intubation.

Cardiovascular changes during videolaparoscopic cholecystectomies and pneumoperitoneum consist basically of increased perioperative SBP, SBP and HR ${ }^{23}$. Our study has shown a statistically significant increase in SBP and DBP in $\mathrm{M}_{5}$ in the dexmedetomidine group, with no change in $\mathrm{HR}$ though. So, EC and EC/MAC were significantly higher in this moment, aiming at maintaining previously established pressure levels.

Other authors ${ }^{24}$ have shown that $8 \mu \mathrm{g} \cdot \mathrm{kg}^{-1}$ remifentanil are able to block vasopressin release during pneumoperitoneum in videolaparoscopic surgeries, what probably helps keeping SBP and DBP levels within acceptable limits for the procedure. Alpha-2 agonists in videolaparoscopic cholecystectomies, more precisely $8 \mu \mathrm{g} \mathrm{kg}^{-1}$ clonidine infusion for one hour, were unable to effectively block plasma vasopressin release during pneumoperitoneum. These data favor our study, which has shown an increase in SBP and DBP in $M_{4}$ and $M_{5}$, probably related to an increase in systemic vascular resistance. It is possible that dexmedetomidine in the dose used, does not block plasma vasopressin and cortisol release, thus allowing an increase in systemic vascular resistance, leading to increased SBP and DPB ${ }^{25}$.

Cell metabolism increase is an important component of surgical stress response and may further compromise patients in bad physical conditions or those submitted to major surgeries. Increased plasma catecholamine concentrations increase cardiac risk and protein catabolism, delaying recovery. Other studies ${ }^{26}$ have shown that laparoscopy leads to a progressive and significant increase in plasma cortisol, epinephrine, norepinephrine, renin and vasopressin concentrations, while other authors evaluating plasma catecholamine levels alone have observed pneumoperitoneum-related increases ${ }^{27-29}$.

Previous studies have shown that opioids are potent inhibitors of the hypophysis-adrenal axis ${ }^{30}$, preventing increases in plasma cortisol, glucose, catecholamine and aldosterone levels during and after cardiopulmonary bypass ${ }^{31,32}$. In healthy volunteers receiving intravenous dexmedetomidine, there has been inhibition of sympathetic autonomous nervous system evaluated by decreased levels of plasma norepinephrine ${ }^{32}$.

In our study, plasma epinephrine and norepinephrine results have shown no statistically significant changes in pneumoperitoneum inflation and deflation response in both groups. So, data suggest that both dexmedetomidine and remifentanil, in the doses used, were equally effective in blocking norepinephrine and epinephrine release triggered by pneumoperitoneum. 
In spite of the demonstration that both drugs are effective in blocking catecholamine release as a consequence of pneumoperitoneum, it is worth stressing that there has been no direct correlation with blood pressure levels during pneumoperitoneum. It is possible that SBP increase was due to vasopressin and cortisol, as already mentioned. To maintain adequate pressure levels during $M_{4}$, a higher sevoflurane EC was used in the dexmedetomidine group. The incidence of postoperative nausea and vomiting was $42 \%$ for group II and $28 \%$ for group I, suggesting that dexmedetomidine may contribute to decrease those symptoms after videolaparoscopic surgeries.

Less patients needed analgesics during the first 6 postoperative hours in GI as compared to II, what may indicate a possible dexmedetomidine analgesic effect.

In conclusion, dexmedetomidine and remifentanil, in the doses used in this study, were effective in blocking hemodynamic response to tracheal intubation, the same not being true during pneumoperitoneum. There has been SBP and DBP increase in the dexmedetomidine group (GI) although epinephrine and norepinephrine levels were not different from the remifentanil group (II).

\section{REFERÊNCIAS - REFERENCES}

01. Anand KJS, Philbin D, Hickey PR - Halothane-morphine compared with high dose sufentanil for anesthesia and postoperative analgesia in neonate cardiac surgery. $\mathrm{N}$ Engl J Med, 1992;326:1-4.

02. Mora CT, Dudek C, Torjman MC - The effects of anaesthetic technique on the hemodynamic response and recovery profile in coronary revascularization patients. Anesth Analg, 1995;81: 900-905.

03. Kono K, Philbin DM, Coggins $\mathrm{CH}$ et al - Renal Function and stress response during halothane or fentanyl anesthesia. Anesth Analg, 1981;60:552-556.

04. Okum GS, Colonna-Romano P, Horrow JC - Vomiting after alfentanil anesthesia: Effect of dosing method. Anesth Analg, 1992; 75:558-562.

05. Sukhani R, Vazquez J, Pappas AL - Recovery after propofol with and without intraoperative fentanyl in patient undergoing ambulatory gynecologic laparoscopy. Anesth Analg, 1996;83: 975-980.

06. Brockmann C, Raasch W, Bastian C - Endocrine stress parameters during TIVA with remifentanil, fentanyl, or sufentanil. Anaesth Intens Noft Schmerzther, 2000;35: 685-691.

07. Flacke JW, Bloor BC, Flacke WE et al - Reduced narcotic requirement fentanyl by clonidine with improved hemodynamic and adrenergic stability in patients undergoing coronary bypass surgery. Anesthesiology, 1987; 67:11-19.

08. Ghignone M, Calvillo O, Quintin L - Anesthesia and hypertension: the effect of clonidine an perioperative hemodynamics and isoflurane requirements. Anesthesiology, 1987;67:3-10.

09. Ghignone M, Quintin L, Duke D et al - Effects of clonidine on narcotics requirement fentanyl and hemodynamics response during induction of anesthesia and endotraqueal intubation. Anesthesiology, 1986;64:36-42.

10. Orko R, Pouttu J, Ghignone M - Effect of clonidine on hemodynamics response to endotraqueal intubation on gastric acidity. Acta Anaesthesiol Scand, 1987;31: 325-329.
11. Maze M, Birch B, Vickery R - Clonidine reduces halothane MAC in rats. Anesthesiology, 1987;67: 868-869.

12. Kaukinen S, Pyykko K - The potentiation of halothane anesthesia by clonidine. Acta Anaesthesiol Scand, 1979;23:107-111.

13. Maze M, Tranquilli W - Alpha 2 adrenoceptor agonists: defining the role in clinical anesthesia. Anesthesiology, 1991;74: 581-605.

14. Aantaa R, Scheinin M - Alpha-2 adrenergic agents in anaesthesia. Acta Anaesthesiol Scand, 1993;37:433-448.

15. Venn RM, Bryant A, Hall GM et al - Effects of dexmedetomidine on adrenocortical function and the cardiovascular, endocrine and inflammatory response in postoperative patients needing sedation in the intensive care unit. $\mathrm{Br} J$ Anaesth, 2000;86: 650-656.

16. Aantaa R, Jaakola M-L, Kallio A et al - Reduction of the minimum alveolar concentration of isoflurane by dexmedetomidine. Anesthesiology, 1997;86:1055-160.

17. Fragen RJ, Fitzgerald PC - Effect of dexmedetomidine on the minimum alveolar concentration (MAC) of sevoflurane in adults age 55 to 70 years. J Clin Anesth, 1999;11:466-470.

18. Scheinin B, Lindgren L, Randell T et al - Dexmedetomidine attenuates sympathoadrenal response to traqueal intubation and reduce the need for thiopentone and peroperative fentanyl. $\mathrm{Br} \mathrm{J}$ Anaesth, 1992;68:126-131.

19. Aho M, Scheinin M, Lehtinen A-M et al - Intramusculary administered dexmedetomidine attenuates hemodynamic and stress hormone response to gynecologic laparoscopy. Anesth Analg, 1992;75: 932-939.

20. Dutta S, Karol MD, Cohen T et al - Effect of dexmedetomidine on propofol requirements in healthy subjects. J Pharm Scienc, 2001;90:172-181.

21. Degani ALG, Cass QB, Vieira PC - Cromatografia um breve ensaio. Química nova escola, 1998;7:21-25.

22. Nunes RR, Cavalcante SL - Influência da dexmedetomidina na concentração expirada do sevoflurano. Avaliação pelo índice bispectral, taxa de supressão e análise espectral da potência do eletroencefalograma. Rev Bras Anestesiol, 2002;52:133-145.

23. Link RE, Dessai K, Hein L - Cardiovascular regulation in mice lacking alfa2-adrenergic receptor subtypes b and c. Science, 1996;273:803-805.

24. Lentschener $\mathrm{C}$, Axler $\mathrm{O}$, Fernandez $\mathrm{H}$ et al - Haemodynamic changes and vasopressin release are not consistently associated with carbon dioxide pneumoperitoneum in humans. Acta Anaesthesiol Scand, 2001;45:527-535.

25. Joris JL, Chiche JD, Canivet JL et al - Hemodynamic changes induced by laparoscopy and their endocrine correlates: effects of clonidine. J Am Coll Cardiol, 1998;32:1389-1396.

26. O'Leary E, Hubbard K, Tormay W et al - Laparoscopic cholecystectomy: haemodynamic and neuroendocrine responses after pneumoperitoneum and changes in position. $\mathrm{Br} \mathrm{J}$ Anaesth, 1996;77:640-644.

27. Joris JL, Chiche JD, Canivet JL et al - Hemodynamics changes induced by laparoscopy and their endocrine correlates: effects of clonidine. J Am Coll Cardiol, 1998;35:1389-1396.

28. Mikami O, Fujise K, Matsumoto S, et al - High intra-abdominal pressure increases plasma cathecolamine concentration during pneumoperitoneum for laparoscopic procedures. Arch Surg, 1998;133:39-43.

29. Myre K, Rostrup M, Buanes T et al - Plasma cathecolamines and haemodynamics changes during pneumoperitoneum. Acta Anaesthesiol Scand, 1998;42: 343-347.

30. Delitalia G, Trainer PJ, Oliva O - Opioid peptide and alpha-adrenoceptor pathways in the regulation of the pituitary adrenal axis in man. J Endocrinol, 1994;141: 163-169. 
31. Stanley TH, Berman L, Green O - Plasma cathecolamine and cortisol responses to fentanyl-oxygen anesthesia for coronary-artery operations. Anesthesiology, 1980;53: 250-255.

32. Sebel PS, Bovil JG, Schellekens APM - Hormonal responses of high-dose fentanyl anesthesia: a study in patients undergoing cardiac surgery. Br J Anaesth, 1981;53:941-945.

\section{RESUMEN}

Chaves TP, Gomes JMA, Pereira FEC, Cavalcante SL, Leitão IMTA, Monte HS, Escalante RD - Evaluación Hemodinámica y Metabólica de la Infusión Continua de Dexmedetomidina y de Remifentanil en Colecistectomia Videolaparoscópica. Estudio Comparativo

JUSTIFICATIVA Y OBJETIVOS: La dexmedetomidina ha sido utilizada para sedación y como coadjuvante en anestesia general. El objetivo de este estudio fue evaluar la respuesta cardiovascular y simpático-adrenal a la intubación traqueal y la insuflación del pneumoperitoneo, comparándola al remifentanil durante anestesia con sevoflurano para colecistectomia videolaparoscópica.

MÉTODO: Fueron incluidos en el estudio 42 pacientes, estado físico ASA I ó II, con edades entre 25 y 55 años, distribuidos aleatoriamente en dos grupos: GI y Gll. La inducción de la anestesia fue realizada con infusión continua de $1 \mu \mathrm{g} . \mathrm{kg}^{-1} \mathrm{de}$ dexmedetomidina (GI) o remifentanil (GII), durante 10 minutos, seguido de propofol y cisatracúrio. La manutención de la anestesia fue realizada con la infusión continua de 0,7 $\mu \mathrm{g} \cdot \mathrm{kg}^{-1} \cdot \mathrm{h}^{-1}$ de dexmedetomidina $00,5 \mu \mathrm{g} \cdot \mathrm{kg}^{-1} \cdot \mathrm{h}^{-1}$ de remifentanil y concentraciones variadas de sevoflurano. Fueron anotadas la PAS, PAD y FC en los momentos: $M_{1}$ - antes del inicio de la infusión inicial de la droga; $M_{2}$ - después del término de la infusión inicial de la droga; $M_{3}$ - después de la intubación orotraqueal: $M_{4}$ - antes del inicio del pneumoperitoneo; $M_{5}$ - después del pneumoperitoneo; $M_{6}$ cinco minutos después desinsuflado el pneumoperitoneo, $M_{7}$ después extubación traqueal. En $M_{4}, M_{5}$ y $M_{6}$ fueron dosadas adrenalina y noradrenalina. La concentración expirada (CE) del sevoflurano, la relación CE/CAM, consumen de sevoflurano fueron registrados en $M_{4}, M_{5}$ y $M_{6}$.

RESULTADOS: Variaciones en la PAS y PAD fueron mayores en el grupo de la dexmedetomidina en $M_{4}$ y $M_{5}$. A FC y los niveles de adrenalina y noradrenalina no presentaron diferencia entre los grupos. $L a C E$ del sevoflurano fue mayor en $M_{4}$ y $M_{6}$ en el Gl, así como la CE/CAM. En el Gl, el consumen de sevoflurano fue mayor y se observó una tendencia para menor consumen de analgésicos y antieméticos.

CONCLUSIONES: En las condiciones de este estudio, la dexmedetomidina inhibió la liberación de catecolaminas durante la intubación orotraqueal y el pneumoperitoneo, sin embargo, no impidió el aumento de la presión arterial en respuesta a la insuflación peritoneal. 